\title{
EFEITO PROLONGADO DE SISTEMAS DE PREPARO DO SOLO COM E SEM CULTIVO DE SOQUEIRA DE CANA CRUA EM ALGUMAS PROPRIEDADES FÍSICAS DO SOLO
}

\section{FÁBIO CAMILOTTI ${ }^{1}$, ITAMAR ANDRIOLI ${ }^{2}$, FÁBIO L. F. DIAS ${ }^{3}$, AILTO A. CASAGRANDE ${ }^{4}$, ALYSSON R. DA SILVA ${ }^{1}$, MIGUEL A. MUTTON ${ }^{5}$, JOSÉ F. CENTURION ${ }^{2}$}

RESUMO: Este trabalho foi conduzido em Latossolo Vermelho distrófico típico, sob cultivo de cana crua, com o objetivo de avaliar o efeito prolongado de sistemas de preparo do solo, com e sem cultivo da soqueira, e épocas de amostragem sobre algumas propriedades físicas do solo. $\mathrm{O}$ delineamento experimental foi o de blocos ao acaso, em esquema de parcelas subsubdivididas, sendo os tratamentos principais quatro sistemas de preparo do solo: (i) controle da soqueira com duas gradagens, subsolagem e mais uma gradagem de nivelamento; (ii) controle da soqueira com herbicida e uma subsolagem; (iii) controle da soqueira com herbicida; (iv) controle da soqueira com herbicida, aração com arado de aivecas e uma gradagem de nivelamento. Os tratamentos secundários foram: com cultivo e sem cultivo da soqueira. Os tratamentos ternários foram as épocas de avaliação: dois dias antes e após a quarta colheita da cultura. A porosidade total, macroporosidade, microporosidade e densidade do solo foram avaliadas, além do perfilhamento e da produtividade da cultura. Após quatro sucessivas colheitas de cana crua, houve redução da macroporosidade com aumento da microporosidade nas camadas abaixo de $10 \mathrm{~cm}$ e aumento da densidade do solo entre 20 e $50 \mathrm{~cm}$. A densidade do solo não foi alterada de modo consistente em função de todos os tratamentos testados. O cultivo da soqueira favoreceu o aumento da macroporosidade com decréscimo na microporosidade, e o efeito inverso foi observado após a colheita. Os sistemas de preparo do solo e de cultivo da soqueira não afetaram o perfilhamento e a produtividade da cultura.

PALAVRAS-CHAVE: densidade do solo, porosidade, Saccharum.

\section{LONG-TERM EFFECT OF SOIL TILLAGE SYSTEMS WITH AND WITHOUT TILLAGE OF GREEN-CANE STUMP IN SOIL PHYSICAL PROPERTIES}

\begin{abstract}
This work was carried out at the field conditions in a Typic Haplustox soil with greencane crop with the objective to evaluate long-term effect of soil tillage systems with and without tillage of stump in soil physical properties. A complete randomized blocks in split-split-plots arrangement of treatments were used. The principal treatments were four soil tillage systems: (i) control of stumps with two harrowing, subsoiling plus a levelling harrowing; (ii) control of stumps with herbicide and a subsoiling; (iii) control of stumps with herbicide; (iv) control of stumps with herbicide, subsoiling plus a levelling harrowing. Secondary treatments were stump tillage systems: with and without stump tillage. The split-split-plot treatments were the evaluation time: before and after the $4^{\text {th }}$ cane harvest. Total porosity, macroporosity, microporosity and bulk density were evaluated, besides the tillering and yield of the crop. After four harvests of green-cane there were reduction of the macroporosity with increase of the microporosity in the soil layers below $10 \mathrm{~cm}$, and an increase of soil bulk density in the layer between 20 and $50 \mathrm{~cm}$. Soil bulk density wasn't altered in a consistent way as affected by all treatments. Stump tillage promoted an increased in the macroporosity with decrease of the microporosity, and the inverse effect was observed after the harvest. Soil tillage system and stump tillage didn't affect both tillering and yield of green-cane.
\end{abstract}

KEYWORDS: bulk density, porosity, Saccharum.

\footnotetext{
${ }^{1}$ Eng $^{\mathrm{o}}$ Agrônomo, Doutorando em Agronomia, Departamento de Solos e Adubos, UNESP, Jaboticabal - SP, Fone (0XX16) 3209-2672, camilotti@bol.com.br. Bolsista da CAPES.

${ }^{2}$ Prof. Adjunto, Departamento de Solos e Adubos, UNESP, Jaboticabal - SP.

${ }^{3}$ Eng ${ }^{\circ}$ Agrônomo, Doutor em Agronomia pela UNESP, Jaboticabal - SP.

${ }^{4}$ Prof. Titular, Departamento de Produção Vegetal, UNESP, Jaboticabal - SP.

${ }^{5}$ Prof. Assistente Doutor, Departamento de Produção Vegetal, UNESP, Jaboticabal - SP.

Recebido pelo Conselho Editorial em: 5-1-2004

Aprovado pelo Conselho Editorial em: 18-2-2005
}

Eng. Agríc., Jaboticabal, v.25, n.1, p.189-198, jan./abr. 2005 


\section{INTRODUÇÃO}

A cana-de-açúcar é estabelecida em sulcos; tratores e colhedoras, quando a colheita é mecanizada, trafegam freqüentemente na entrelinha da cultura. A pressão exercida pelas máquinas e implementos sobre o solo pode causar a compactação do mesmo. Como conseqüência, há alteração na densidade do solo decorrente da modificação da sua estrutura (KLEIN \& LIBARDI, 2002), o que afeta as propriedades físico-hídricas fundamentais, como porosidade de aeração, retenção de água, disponibilidade de água às plantas e a resistência do solo à penetração (TORMENA et al., 1998), resultando, em última instância, no declínio da produtividade da lavoura. BORGES et al. (1999) acrescentam que a compactação proporciona a redução linear da porosidade total e do espaço de aeração do solo.

Para descompactar o solo durante o ciclo da cultura, emprega-se o escarificador no cultivo da entrelinha da soqueira, o que altera a densidade do solo. CERQUEIRA LUZ (1989) observou reduções significativas na densidade da camada superficial do solo pelo cultivo da soqueira em sistema de colheita de cana queimada. Por outro lado, alguns estudos (ORLANDO FILHO et al., 1994; ORLANDO FILHO et al., 1998) têm indicado poucas respostas em produção para utilização de cultivo em áreas de colheita mecanizada, sem a prévia despalha a fogo.

Outra oportunidade para a descompactação do solo é justamente na reforma do canavial. Nessa ocasião, empregam-se implementos que efetivamente descompactam o solo, a exemplo do subsolador, que atua em até subsuperfície. Pesquisas envolvendo o preparo de solo em áreas de cana-de-açúcar colhida com prévia despalha a fogo têm sido realizadas há algum tempo (FERNANDES et al., 1983; BARBIERI et al., 1997), e, recentemente, trabalhos dessa natureza foram conduzidos em áreas de cana crua. Testando alguns métodos de preparo de solo, DIAS (2001) constatou que o preparo reduzido em área de cana crua proporcionou incrementos na produtividade da cultura.

Porém, o próprio sistema de colheita tem afetado as propriedades físicas do solo. CEDDIA et al. (1999) constataram, comparativamente, aumento na densidade do solo em sistema de colheita de cana queimada e maior porcentagem de poros totais na camada de $0-20 \mathrm{~cm}$ em sistema de colheita de cana crua. Isso é indicativo de que estudos sobre o comportamento das propriedades do solo, em função do manejo do mesmo, devem levar em consideração o sistema adotado de colheita da cana-de-açúcar.

Não está bem documentado o efeito a longo prazo de sistemas de preparo de solo, combinados com a realização ou não do cultivo de soqueiras de cana-de-açúcar, sobre algumas propriedades do solo. Assim, o objetivo deste trabalho foi o de avaliar o efeito prolongado de sistemas de preparo de solo, com e sem cultivo de soqueiras, e épocas de amostragem, antes e após a quarta colheita de canacrua, sobre a macroporosidade, microporosidade e densidade do solo. Procurou-se analisar, ainda, o perfilhamento e a produtividade da cultura.

\section{MATERIAL E MÉTODOS}

O experimento foi realizado na Fazenda Ouro da Açucareira Corona, Usina Bonfim, no município de Guariba - SP, com latitude de 20³0'S, longitude de 48¹3'W e altitude média de $600 \mathrm{~m}$. Utilizou-se da cultivar de cana-de-açúcar RB855536 e o solo da área foi classificado como Latossolo Vermelho distrófico típico, segundo EMBRAPA (1999). A cultura foi implantada em dezembro de 1997, sendo, nessa área, utilizado o sistema de colheita de cana crua há quatro anos.

O delineamento experimental foi o de blocos ao acaso, em parcelas subsubdivididas, com quatro repetições, em que os tratamentos principais se constituíram de diferentes sistemas de preparo do solo; os secundários de dois sistemas de cultivo e os tratamentos ternários de duas épocas de avaliação das propriedades do solo. 
As parcelas foram constituídas de seis sulcos com $60 \mathrm{~m}$, espaçadas em 1,40 m, sendo divididas em duas subparcelas, de seis sulcos com $30 \mathrm{~m}$, sendo adotados quatro sistemas de preparo. O primeiro, denominado de Convencional $(\mathrm{GP}+\mathrm{S})$, constou de controle da soqueira com duas gradagens (grade aradora com 16 discos recortados de $80 \mathrm{~mm}$ ), subsolagem aproximadamente até $60 \mathrm{~cm}$ (subsolador com cinco hastes e ponteiras aletadas, equipado com os acessórios disco corta-palha e rolo destorroador) mais uma gradagem de nivelamento do terreno (grade niveladora com 24 discos de 70 $\mathrm{mm}$ ); o segundo, denominado de Subsolador (S), constou de controle da soqueira com herbicida (glifosate) e uma subsolagem aproximadamente até $60 \mathrm{~cm}$ (subsolador com cinco hastes e ponteiras aletadas, equipado com os acessórios disco corta-palha e rolo destorroador); o terceiro, denominado de Cultivo Mínimo (CM), constou de controle da soqueira com herbicida (glifosate), e o quarto, denominado de Aração com Arado Aivecas (AA), constou de controle da soqueira com herbicida (glifosate), aração (arado com quatro aivecas) e uma gradagem de nivelamento (grade niveladora com 24 discos de $70 \mathrm{~mm})$.

As subparcelas associadas com os sistemas de preparo de solo foram os sistemas de cultivo da soqueira, constituídos de: operações de cultivo com um cultivador de duas hastes parabólicas e ponteiras não-aletadas, equipado com acessório disco corta-palha e ausência de disco destorroador, atuando à profundidade de aproximadamente $30 \mathrm{~cm}$ (Com Cultivo) e adubação localizada por cima da palha com auxílio de adubadora-cultivadora (Sem Cultivo).

As épocas referentes às operações de implantação, condução e colheita da cana-de-açúcar estão apresentadas na Tabela 1.

TABELA 1. Época de realização das operações referentes à implantação, condução e colheita da cultura.

\begin{tabular}{ll}
\hline Época & \multicolumn{1}{c}{ Operações } \\
\hline dezembro de 1997 & Aplicação de calcário. \\
janeiro de 1998 & Aplicação de herbicida conforme os tratamentos e preparo de solo. \\
abril de 1998 & Sulcação, adubação, plantio de mudas e aplicação de cupinicida. \\
janeiro de 2002 & Cultivo da terceira soqueira. \\
setembro de 2002 & Colheita da cana-soca de quarto corte. \\
\hline
\end{tabular}

A calagem constou da aplicação em área total de 1,5 t ha ${ }^{-1}$ de calcário dolomítico (PRNT 75\%) para elevar a saturação de bases a $60 \%$ (RAIJ et al., 1996). Aplicaram-se $2,4 \mathrm{~L} \mathrm{ha}^{-1}$ de glifosate no tratamento SP e 1,4 $\mathrm{L} \mathrm{ha}^{-1}$ nos tratamentos S e AA. Em todos os tratamentos, foi realizada uma sulcação com sulcador com ponteiras aletadas de duas linhas para abertura de sulcos com $25 \mathrm{~cm}$ de profundidade, momento em que também se fez a adubação de plantio com 1.000 L ha ${ }^{-1}$ da fórmula 2,512-06. Realizou-se o plantio das mudas de cana-de-açúcar, procurando-se manter uma densidade de 15 gemas por metro de sulco, simultaneamente à aplicação $5 \mathrm{~L} \mathrm{ha}^{-1}$ de cupinicida. Na ocasião do cultivo da soqueira (correspondente à subparcela), foram aplicados $570 \mathrm{~kg} \mathrm{ha}^{-1}$ da fórmula 14-07-28 em cobertura.

A avaliação de perfilhamento da cultura foi realizada em abril de 2002, contando-se o número de perfilhos em $20 \mathrm{~m}$ de cada uma das quatro linhas centrais da subparcela. A produtividade da cana-deaçúcar foi estimada segundo SILVA (1989), cuja avaliação no campo foi feita 15 dias antes da colheita da cultura. Contou-se o número de colmos em $20 \mathrm{~m}$ de cada uma das quatro linhas centrais da subparcela, totalizando $80 \mathrm{~m}$. Foram colhidos, despontados e pesados 10 colmos de cada uma das quatro linhas centrais da subparcela.

As amostras indeformadas, utilizando-se de anéis volumétricos, foram tiradas nas profundidades de $0-10 ; 10-20 ; 20-30 ; 30-40$ e 40-50 cm no meio da entrelinha da cultura, 60 dias antes do preparo do 
solo, dois dias antes e após a colheita da cultura. A porosidade total foi obtida pela diferença entre a massa do solo saturado e a massa do solo seco em estufa a $110^{\circ} \mathrm{C}$ durante $24 \mathrm{~h}$ (EMBRAPA, 1997). A microporosidade do solo foi determinada pelo método da mesa de tensão com uma coluna de água de $60 \mathrm{~cm}$ de altura, segundo EMBRAPA (1997). Pela diferença entre a porosidade total e a microporosidade, obteve-se a macroporosidade. A densidade do solo foi calculada pela relação entre a massa seca a $110{ }^{\circ} \mathrm{C}$ durante $24 \mathrm{~h}$ da amostra de solo do cilindro volumétrico e o volume do mesmo cilindro (EMBRAPA, 1997).

Os resultados da porosidade total, macro e microporosidade e densidade do solo antes da instalação do experimento estão apresentados na Tabela 2.

TABELA 2. Porosidade total (PT), microporosidade (Micro), macroporosidade (Macro) e densidade do solo (Ds) antes da instalação do experimento.

\begin{tabular}{ccccc}
\hline Profundidade $(\mathrm{cm})$ & PT & Micro & Macro & Ds \\
\cline { 2 - 5 } $0-10$ & 59,28 & 50,26 & 9,02 & $\mathrm{~kg} \mathrm{~m}^{-3}$ \\
$10-20$ & 60,03 & 38,39 & 21,63 & 1,70 \\
$20-30$ & 60,08 & 37,05 & 23,04 & 1,65 \\
$30-40$ & 62,14 & 42,64 & 19,50 & 1,62 \\
$40-50$ & 59,28 & 37,96 & 21,64 & 1,53 \\
\end{tabular}

\section{RESULTADOS E DISCUSSÃO}

Os resultados das análises de variância para as propriedades físicas do solo analisadas nas camadas de 0-10 e 10-20 cm; 20-30 e 30-40 cm, e 40-50 cm estão apresentados nas Tabelas 3; 6 e 7, respectivamente.

O tratamento Cultivo Mínimo (CM) apresentou maiores valores de densidade na camada de 10$20 \mathrm{~cm}$ em relação à Aração com Arado de Aivecas (AA) (Tabela 3), o que é explicado pelo efeito prolongado da descompactação da camada superficial do solo promovida por tal aração antes da implantação da cultura.

Pelas Tabelas 3; 6 e 7, observa-se que não houve efeito significativo dos sistemas de cultivo e épocas de avaliação na densidade do solo, discordando dos resultados de CERQUEIRA LUZ (1989), o qual constatou que o emprego do cultivo da soqueira reduziu a densidade do solo em sistema de cana queimada, e o tráfego de máquinas durante a colheita, ao contrário, aumentou os valores dessa propriedade física. Porém, em sistema de colheita de cana crua, VASCONCELOS (2002) não encontrou aumento da densidade do solo na camada de $0-10 \mathrm{~cm}$ pelo efeito do tráfego de máquinas na ocasião da colheita, o que se confirma no presente estudo.

Considerando os valores para a densidade do solo antes da implantação da cultura (Tabela 2), observa-se que a mesma foi pouco alterada nas camadas de 0-10 e 10-20 cm após quatro anos de sucessão de ciclos da cana-de-açúcar em regime de colheita de cana crua (Tabela 3). Por outro lado, nas camadas abaixo de $20 \mathrm{~cm}$, houve aumento na densidade do solo após o quarto corte da cultura, o que significa haver efeito de compactação do solo em subsuperfície devido ao manejo mecanizado da cultura.

O valor da macroporosidade na camada do solo de $0-10 \mathrm{~cm}$ para tratamento AA foi maior em relação ao Convencional $(\mathrm{GP}+\mathrm{S})$, resultado que reflete diretamente no aumento da porosidade total (Tabela 3). Isso pode ser explicado tendo em vista que o preparo convencional do solo desagrega mais as partículas do que a mobilização do mesmo pelo arado de aivecas, o que, provavelmente, resultou no menor valor para a macroporosidade no primeiro sistema de preparo. 
TABELA 3. Porosidade total (PT), macroporosidade (Macro), microporosidade (Micro) e densidade do solo (Ds) nas profundidades de 0-10 cm e 10-20 cm, em função dos sistemas de preparo do solo (SP), sistemas de cultivo (C) e épocas de amostragem do solo (E).

\begin{tabular}{|c|c|c|c|c|c|c|c|c|}
\hline \multirow{2}{*}{ Fonte de Variação } & \multicolumn{4}{|c|}{$0-10 \mathrm{~cm}$} & \multicolumn{4}{|c|}{$10-20 \mathrm{~cm}$} \\
\hline & PT & Micro & Macro & Ds & $\mathrm{PT}$ & Micro & Macro & Ds \\
\hline & & $-\%$ & 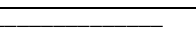 & $\mathrm{kg} \mathrm{dm}^{-3}$ & & $\%$ & & $\mathrm{~kg} \mathrm{dm}^{-3}$ \\
\hline \multicolumn{9}{|l|}{ SP } \\
\hline$\overline{\mathrm{GP}}+\mathrm{S}^{(1)}$ & $53,27 \mathrm{~B}^{(5)}$ & 44,90 & $8,37 \mathrm{~B}$ & 1,69 & 53,61 & $42,39 \mathrm{~B}$ & 11,27 & $1,69 \mathrm{AB}$ \\
\hline$S^{(2)}$ & $54,02 \mathrm{AB}$ & 44,64 & $11,81 \mathrm{AB}$ & 1,64 & 53,84 & $42,18 \mathrm{AB}$ & 11,64 & $1,68 \mathrm{AB}$ \\
\hline $\mathrm{CM}^{(3)}$ & $54,64 \mathrm{AB}$ & 44,48 & $10,19 \mathrm{AB}$ & 1,63 & 53,83 & $42,97 \mathrm{AB}$ & 10,81 & $1,71 \mathrm{~A}$ \\
\hline $\mathrm{AA}^{(4)}$ & $57,29 \mathrm{~A}$ & 44,47 & $12,98 \mathrm{~A}$ & 1,65 & 54,83 & $43,51 \mathrm{~A}$ & 11,94 & $1,66 \mathrm{~B}$ \\
\hline \multicolumn{9}{|l|}{$\mathrm{C}$} \\
\hline Com Cultivo & 54,92 & 44,19 & 11,82 & 1,64 & 54,23 & $40,57 \mathrm{~B}$ & $13,79 \mathrm{~A}$ & 1,68 \\
\hline Sem Cultivo & 54,56 & 44,92 & 9,82 & 1,66 & 53,84 & $44,98 \mathrm{~A}$ & $8,85 \mathrm{~B}$ & 1,69 \\
\hline \multicolumn{9}{|l|}{$\mathbf{E}$} \\
\hline Antes da $4^{\mathrm{a}}$ colheita & $53,69 \mathrm{~B}$ & $41,74 \mathrm{~B}$ & $12,58 \mathrm{~A}$ & 1,64 & 54,23 & 42,49 & $12,21 \mathrm{~A}$ & 1,68 \\
\hline Após a $4^{\mathrm{a}}$ colheita & $56,41 \mathrm{~A}$ & $47,41 \mathrm{~A}$ & $9,05 \mathrm{~B}$ & 1,66 & 53,54 & 43,06 & $10,44 \mathrm{~B}$ & 1,69 \\
\hline Causa de variação & & & & Teste & & & & \\
\hline $\mathrm{SP}$ & $4,12 *$ & $1,19 \mathrm{NS}$ & $4,02 *$ & $2,00 \mathrm{NS}$ & $2,28 \mathrm{NS}$ & $4,81^{*}$ & $0,34 \mathrm{NS}$ & $3,39 \mathrm{NS}$ \\
\hline $\mathrm{C}$ & $0,44 \mathrm{NS}$ & $0,10 \mathrm{NS}$ & $2,55 \mathrm{NS}$ & $1,01 \mathrm{NS}$ & $1,10 \mathrm{NS}$ & $120,99 * *$ & $48,46^{* *}$ & $1,80 \mathrm{NS}$ \\
\hline $\mathrm{E}$ & $5,02 *$ & $59,40 * *$ & $15,99 * *$ & $2,91 \mathrm{NS}$ & $2,37 \mathrm{NS}$ & $0,73 \mathrm{NS}$ & $2,27 *$ & $0,64 \mathrm{NS}$ \\
\hline SP $\times C$ & $0,23 \mathrm{NS}$ & $1,22 \mathrm{NS}$ & $1,06 \mathrm{NS}$ & $0,21 \mathrm{NS}$ & $0,27 \mathrm{NS}$ & $8,09 * *$ & $1,26 \mathrm{NS}$ & $1,09 \mathrm{NS}$ \\
\hline SP $x \mathrm{E}$ & $2,10 \mathrm{NS}$ & $1,69 \mathrm{NS}$ & $1,29 \mathrm{NS}$ & $1,92 \mathrm{NS}$ & $0,54 \mathrm{NS}$ & $0,37 \mathrm{NS}$ & $0,38 \mathrm{NS}$ & $0,39 \mathrm{NS}$ \\
\hline $\mathrm{C} \times \mathrm{E}$ & $12,82 * *$ & $78,99 * *$ & $9,74 * *$ & $3,61 \mathrm{NS}$ & $2,19 \mathrm{NS}$ & $14,48 * *$ & $0,11 \mathrm{NS}$ & $1,23 \mathrm{NS}$ \\
\hline $\mathrm{SP} \times \mathrm{C} \times \mathrm{E}$ & $1,06 \mathrm{NS}$ & $1,37 \mathrm{NS}$ & $3,85^{*}$ & $3,46 \mathrm{NS}$ & $2,41 \mathrm{NS}$ & $2,93 \mathrm{NS}$ & $2,60 \mathrm{NS}$ & $1,08 \mathrm{NS}$ \\
\hline$\overline{\mathrm{CV}^{(7)} \mathrm{SP}(\%)}$ & 7,92 & 4,43 & 38,50 & 4,43 & 5,54 & 3,95 & 30,73 & 2,99 \\
\hline $\mathrm{CV}^{(7)} \mathrm{C}(\%)$ & 11,37 & 5,51 & 49,48 & 5,13 & 5,09 & 3,53 & 25,39 & 2,48 \\
\hline $\mathrm{CV}^{(7)} \mathrm{E}(\%)$ & 8,66 & 5,99 & 33,82 & 3,91 & 6,29 & 3,42 & 31,30 & 3,81 \\
\hline
\end{tabular}

Pela Tabela 4, observa-se que o cultivo da soqueira favoreceu o aumento na macroporosidade com a redução da microporosidade na amostragem feita antes da $4^{\underline{a}}$ colheita $(0-10 \mathrm{~cm})$, corroborando os resultados de CASAGRANDE (1973). No entanto, esse aumento na macroporosidade foi suprimido após a colheita da cultura (Tabela 4), provavelmente pelo efeito do tráfego de máquinas. GUPTA et al. (1989) explicaram que os macroporos são sensíveis às pressões exercidas sobre o solo, o que pode justificar a redução na macroporosidade após a colheita realizada com máquinas e implementos que compactam o solo.

TABELA 4. Porosidade total (PT), microporosidade (Micro) e macroporosidade (Macro) na camada de $0-10 \mathrm{~cm}$ do solo em função das épocas de amostragem e sistemas de cultivo da soqueira. Valores em porcentagem.

Sistema de Cultivo Época de Amostragem

\begin{tabular}{|c|c|c|}
\hline & Antes da $4^{\mathrm{a}}$ Colheita & Depois da 4- Colheita \\
\hline & \multicolumn{2}{|r|}{ 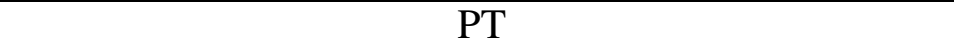 } \\
\hline \multirow{3}{*}{$\begin{array}{l}\text { Com cultivo } \\
\text { Sem cultivo }\end{array}$} & $51.81 \mathrm{Ab}$ & $58.70 \mathrm{Aa}$ \\
\hline & $55,01 \mathrm{Aa}$ & $53,42 \mathrm{Ba}$ \\
\hline & \multicolumn{2}{|c|}{ Micro } \\
\hline \multirow{3}{*}{$\begin{array}{l}\text { Com cultivo } \\
\text { Sem cultivo }\end{array}$} & $39.12 \mathrm{Bb}$ & $50,19 \mathrm{Aa}$ \\
\hline & $44,86 \mathrm{Aa}$ & $44,07 \mathrm{Ba}$ \\
\hline & \multicolumn{2}{|c|}{ Macro } \\
\hline Com cultivo & $15,16 \mathrm{Aa}$ & $8.64 \mathrm{Ab}$ \\
\hline Sem cultivo & $10,16 \mathrm{Ba}$ & $9,35 \mathrm{Aa}$ \\
\hline
\end{tabular}

Letras maiúsculas comparam sistemas de cultivo e as minúsculas épocas de amostragem, pelo teste de Tukey $(\mathrm{P}<0,05)$. 
Para as camadas 10-20; 20-30, e 30-40 cm, houve aumento da macroporosidade pelo cultivo da soqueira (Tabelas 3 e 6). A microporosidade aumentou na camada de 10-20 cm quando não foi efetuado o cultivo da soqueira e também quando se empregou a aração com arado de aivecas associado ao cultivo da soqueira (Tabela 5). Já para as camadas de 30-40 e 40-50 cm, a ausência do cultivo da soqueira resultou no aumento da microporosidade na avaliação feita após a quarta colheita, o que não ocorreu para a camada de $10-20 \mathrm{~cm}$ (Tabela 8).

Tendo como base os valores da Tabela 2, após quatro anos de cultivo de cana-de-açúcar, observou-se que houve redução da macroporosidade e conseqüente aumento da microporosidade nas camadas abaixo de $10 \mathrm{~cm}$, resultados ligados ao manejo da cultura com alto grau de mecanização, o que pode ser atribuído à compactação do solo.

TABELA 5. Microporosidade (\%) na camada de $10-20 \mathrm{~cm}$ do solo nas áreas com e sem cultivo da soqueira em função dos sistemas de preparo de solo.

\begin{tabular}{lcc}
\hline Sistema de Preparo & \multicolumn{2}{c}{ Sistema de Cultivo da Soqueira } \\
\cline { 2 - 3 } & Com Cultivo & Sem Cultivo \\
\hline $\mathrm{GP}+\mathrm{S}^{(1)}$ & $40,00 \mathrm{Bb}$ & $44,55 \mathrm{Aa}$ \\
$\mathrm{S}^{(2)}$ & $39,48 \mathrm{Bb}$ & $45,16 \mathrm{Aa}$ \\
$\mathrm{CM}^{(3)}$ & $39,65 \mathrm{Bb}$ & $45,06 \mathrm{Aa}$ \\
$\mathrm{AA}^{(4)}$ & $43,66 \mathrm{Aa}$ & $44,67 \mathrm{Aa}$ \\
${ }^{(1)} \mathrm{GP}+\mathrm{S}=$ duas gradagens pesadas + subsolagem + nivelamento; ${ }^{(2)} \mathrm{S}=$ herbicida + subsolagem; ${ }^{(3)} \mathrm{CM}=$ cultivo mínimo; ${ }^{(4)} \mathrm{AA}$ \\
$=$ herbicida + arado de aivecas; ${ }^{(5)}$ médias seguidas pela mesma letra, maiúscula na coluna e minúscula na linha, não diferem entre \\
si, pelo teste de Tukey $(\mathrm{P}<0,05)$.
\end{tabular}

TABELA 6. Porosidade total (PT), macroporosidade (Macro), microporosidade (Micro) e densidade do solo (Ds) nas profundidades de $20-30 \mathrm{~cm}$ e $30-40 \mathrm{~cm}$, em função dos sistemas de preparo do solo (SP), sistemas de cultivo (C) e épocas de amostragem do solo (E).

\begin{tabular}{|c|c|c|c|c|c|c|c|c|}
\hline \multirow{2}{*}{ Tratamentos } & \multicolumn{4}{|c|}{$20-30 \mathrm{~cm}$} & \multicolumn{4}{|c|}{$30-40 \mathrm{~cm}$} \\
\hline & PT & Micro & Macro & Ds & PT & Micro & Macro & Ds \\
\hline & & $-\%$ & & $\mathrm{~kg} \mathrm{dm}^{-3}$ & & $-\%$ & & $\mathrm{~kg} \mathrm{dm}^{-3}$ \\
\hline \multicolumn{9}{|l|}{$\overline{\mathbf{S P}}$} \\
\hline$\overline{\mathrm{GP}}+\mathrm{S}^{(1)}$ & 54,87 & 44,94 & 9,92 & 1,70 & $56,58 \mathrm{~A}$ & 43,59 & 12,78 & 1,63 \\
\hline$S^{(2)}$ & 54,38 & 42,97 & 10,64 & 1,69 & $54,12 \mathrm{~B}$ & 43,67 & 10,45 & 1,67 \\
\hline $\mathrm{CM}^{(3)}$ & 53,49 & 46,41 & 8,17 & 1,72 & $53,86 \mathrm{~B}$ & 44,97 & 10,82 & 1,67 \\
\hline $\mathrm{AA}^{(4)}$ & 53,49 & 43,54 & 10,10 & 1,67 & $54,12 \mathrm{AB}$ & 44,25 & 11,39 & 1,63 \\
\hline \multicolumn{9}{|c|}{ 年, } \\
\hline Com Cultivo & 54,45 & 43,59 & $10,90 \mathrm{~A}$ & 1,69 & 55,62 & $42,72 \mathrm{~B}$ & $12,95 \mathrm{~A}$ & 1,65 \\
\hline Sem Cultivo & 53,69 & 45,32 & $8,53 \mathrm{~B}$ & 1,70 & 53,76 & $44,72 \mathrm{~A}$ & $9,80 \mathrm{~B}$ & 1,65 \\
\hline \multicolumn{9}{|l|}{$\mathbf{E}$} \\
\hline Antes da $4^{\mathrm{a}}$ colheita & 54,19 & 44,59 & 10,15 & 1,69 & 53,77 & $42,62 \mathrm{~B}$ & 11,28 & 1,65 \\
\hline Após a $4^{\mathrm{a}}$ colheita & 53,94 & 44,32 & 9,28 & 1,70 & 55,59 & $44,79 \mathrm{~A}$ & 11,45 & 1,65 \\
\hline Causa de variação & & & & $-\mathrm{Te}$ & & & & \\
\hline $\mathrm{SP}$ & $2,23 \mathrm{NS}$ & $1,70 \mathrm{NS}$ & $3,52 \mathrm{NS}$ & $1,75 \mathrm{NS}$ & $5,83^{*}$ & $0,96 \mathrm{NS}$ & $2,39 \mathrm{NS}$ & $1,32 \mathrm{NS}$ \\
\hline $\mathrm{C}$ & $1,31 \mathrm{NS}$ & $1,32 \mathrm{NS}$ & $48,58 * *$ & $0,13 \mathrm{NS}$ & $1,94 \mathrm{NS}$ & $6,24 *$ & $5,75^{*}$ & $0,15 \mathrm{NS}$ \\
\hline $\mathrm{E}$ & $0,60 \mathrm{NS}$ & $0,29 \mathrm{NS}$ & $1,08 \mathrm{NS}$ & $0,19 \mathrm{NS}$ & $3,28 \mathrm{NS}$ & $4,54 *$ & $0,03 \mathrm{NS}$ & $0,09 \mathrm{NS}$ \\
\hline SP x C & $1,20 \mathrm{NS}$ & $1,36 \mathrm{NS}$ & $1,94 \mathrm{NS}$ & $0,64 \mathrm{NS}$ & $0,22 \mathrm{NS}$ & $1,28 \mathrm{NS}$ & $0,41 \mathrm{NS}$ & $0,45 \mathrm{NS}$ \\
\hline SP x E & $0,72 \mathrm{NS}$ & $1,39 \mathrm{NS}$ & $0,79 \mathrm{NS}$ & $0,11 \mathrm{NS}$ & $1,53 \mathrm{NS}$ & $1,59 \mathrm{NS}$ & $0,61 \mathrm{NS}$ & $0,31 \mathrm{NS}$ \\
\hline $\mathrm{C} \times \mathrm{E}$ & $0,06 \mathrm{NS}$ & $0,99 \mathrm{NS}$ & $0,14 \mathrm{NS}$ & $0,01 \mathrm{NS}$ & $2,86 \mathrm{NS}$ & $5,39 *$ & $0,05 \mathrm{NS}$ & $2,76 \mathrm{NS}$ \\
\hline SP x C x E & $0,67 \mathrm{NS}$ & $0,51 \mathrm{NS}$ & $0,36 \mathrm{NS}$ & $2,57 \mathrm{NS}$ & $2,05 \mathrm{NS}$ & $3,41^{*}$ & $1,57 \mathrm{NS}$ & $1,37 \mathrm{NS}$ \\
\hline$\overline{\mathrm{CV}^{(7)} \mathrm{SP}(\%)}$ & 4,53 & 7,59 & 25,34 & 3,16 & 8,59 & 26,20 & 4,67 & 4,53 \\
\hline $\mathrm{CV}^{(7)} \mathrm{C}(\%)$ & 7,11 & 11,57 & 23,04 & 4,50 & 7,32 & 46,05 & 5,40 & 7,11 \\
\hline $\mathrm{CV}^{(7)} \mathrm{E}(\%)$ & 5,56 & 8,89 & 36,77 & 3,03 & 9,38 & 35,65 & 3,65 & 5,56 \\
\hline
\end{tabular}


TABELA 7. Porosidade total (PT), macroporosidade (Macro), microporosidade (Micro) e densidade do solo (Ds) na profundidade de 40-50 cm, em função dos sistemas de preparo do solo (SP), sistemas de cultivo (C) e épocas de amostragem do solo.

\begin{tabular}{|c|c|c|c|c|}
\hline Fonte de Variação & PT & Micro & Macro & Ds \\
\hline & & $\%-$ & $\overline{-}$ & $\mathrm{kg} \mathrm{dm}^{-3}$ \\
\hline \multicolumn{5}{|l|}{$\overline{\mathbf{S P}}$} \\
\hline$\overline{\mathrm{GP}}+\mathrm{S}^{(1)}$ & 55,15 & 42,84 & 12,00 & 1,60 \\
\hline$S^{(2)}$ & 53,73 & 42,50 & 11,23 & 1,57 \\
\hline $\mathrm{CM}^{(3)}$ & 55,84 & 43,38 & 12,00 & 1,60 \\
\hline $\mathrm{AA}^{(4)}$ & 57,29 & 44,43 & 13,28 & 1,57 \\
\hline \multicolumn{5}{|l|}{ C } \\
\hline Com Cultivo & 55,92 & 41,97 & 12,99 & 1,59 \\
\hline Sem Cultivo & 55,44 & 43,81 & 11,33 & 1,58 \\
\hline \multicolumn{5}{|l|}{$\mathbf{E}$} \\
\hline Antes da $4^{\mathrm{a}}$ colheita & 55,21 & $43,52 \mathrm{~B}^{(5)}$ & 11,27 & 1,57 \\
\hline Após a 4ª colheita & 55,79 & $46,26 \mathrm{~A}$ & 12,99 & 1,60 \\
\hline & \multicolumn{4}{|c|}{- Teste $\mathrm{F}^{(6)}$} \\
\hline SP & $3,87 \mathrm{NS}$ & $2,79 \mathrm{NS}$ & $1,38 \mathrm{NS}$ & $0,76 \mathrm{NS}$ \\
\hline $\mathrm{C}$ & $0,27 \mathrm{NS}$ & $1,52 \mathrm{NS}$ & $4,35 \mathrm{NS}$ & $0,79 \mathrm{NS}$ \\
\hline E & $8,67 \mathrm{NS}$ & $66,08^{* *}$ & $2,56 \mathrm{NS}$ & $1,91 \mathrm{NS}$ \\
\hline $\mathrm{SP} \times \mathrm{C}$ & $1,53 \mathrm{NS}$ & $0,46 \mathrm{NS}$ & $1,64 \mathrm{NS}$ & $1,75 \mathrm{NS}$ \\
\hline SP $x \mathrm{E}$ & $1,74 \mathrm{NS}$ & $2,05 \mathrm{NS}$ & $0,97 \mathrm{NS}$ & $1,48 \mathrm{NS}$ \\
\hline $\mathrm{C} \times \mathrm{E}$ & $1,47 \mathrm{NS}$ & $17,81 * *$ & $2,05 \mathrm{NS}$ & $0,50 \mathrm{NS}$ \\
\hline SP x C x E & $0,31 \mathrm{NS}$ & $0,86 \mathrm{NS}$ & $0,83 \mathrm{NS}$ & $1,61 \mathrm{NS}$ \\
\hline $\mathrm{CV}^{(7)} \mathrm{SP}(\%)$ & 5,92 & 5,91 & 25,15 & 4,30 \\
\hline $\mathrm{CV}^{(7)} \mathrm{C}(\%)$ & 6,73 & 9,86 & 26,07 & 4,98 \\
\hline $\mathrm{CV}^{(7)} \mathrm{E}(\%)$ & 8,45 & 5,76 & 30,74 & 4,90 \\
\hline
\end{tabular}

TABELA 8. Microporosidade nas camadas de 10-20; 30-40 e 40-50 cm em função das épocas de amostragem e sistemas de cultivo da soqueira. Valores em porcentagem.

Sistema de Cultivo

\begin{tabular}{ccc}
\multicolumn{3}{c}{ Época de Amostragem } \\
\hline Antes da $4^{\mathrm{a}}$ colheita & \multicolumn{2}{c}{ Depois da $4^{\mathrm{a}}$ colheita } \\
\hline \multicolumn{3}{c}{$10-20 \mathrm{~cm}$} \\
\hline $39,85 \mathrm{Bb}$ & $41,55 \mathrm{Ba}$ \\
$45,39 \mathrm{Aa}$ & & $44,31 \mathrm{Ab}$ \\
\hline \multicolumn{3}{c}{$30-40 \mathrm{~cm}$} \\
\hline $42,80 \mathrm{Aa}$ & $42,61 \mathrm{Ba}$ \\
$42,43 \mathrm{Ab}$ & $46,99 \mathrm{Aa}$ \\
\hline \multicolumn{3}{c}{} \\
\hline $41,41 \mathrm{Ab}$ & $40-50 \mathrm{~cm}$ \\
$40,10 \mathrm{Ab}$ & & $43,85 \mathrm{Ba}$ \\
\hline
\end{tabular}

Com cultivo

Sem cultivo

Com cultivo

Sem cultivo

Com cultivo

Sem cultivo

Época de Amostragem

Letras maiúsculas comparam sistemas de cultivo e as minúsculas épocas de amostragem, pelo teste de Tukey $(\mathrm{P}<0,05)$.

Observa-se que o número de perfilhos e a produtividade da cana-de-açúcar (Tabela 9) não foram influenciados pelos sistemas de preparado do solo e cultivo da soqueira. MUTTON (1983) encontrou 
acréscimos na produtividade, nos primeiros ciclos de cana queimada, pelo controle da soqueira com herbicida (glifosate) no preparo de solo. Por outro lado, CEDDIA et al. (1999) encontraram produtividade média de $46 \mathrm{t} \mathrm{ha}^{-1}$ para a quarta colheita de cana crua (cv. RB739735) cultivada em Podzólico Amarelo. Essa baixa produtividade assemelha-se às encontradas no presente experimento.

A baixa produtividade obtida na quarta colheita de cana-crua neste experimento é explicada, em parte, pelos seguintes fatores: (i) a densidade do solo avaliada após quatro anos de cultivo foi maior que $1,45 \mathrm{~kg} \mathrm{~m}^{-3}$, o que prejudica a absorção de nutrientes pela planta (FERNANDES et al., 1983); (ii) a macroporosidade inferior a 15\%, o que segundo BARBIERI (1995) e TROUSE JUNIOR (1965), citado por DIAS (2002), prejudica o desenvolvimento do sistema radicular da cana-de-açúcar; (iii) infestação da cultura com cigarrinha das raízes em sistema de colheita de cana crua.

TABELA 9. Efeitos dos sistemas de preparo de solo (SP) e de cultivo (C) da soqueira sobre perfilhamento e produtividade da cana-de-açúcar.

\begin{tabular}{|c|c|c|}
\hline Fonte de Variação & Perfilhos $\mathrm{m}^{-1}$ & Produtividade $\left(\mathrm{t} \mathrm{ha}^{-1}\right)$ \\
\hline \multicolumn{3}{|l|}{ SP } \\
\hline $\mathrm{GP}+\mathrm{S}^{(1)}$ & 13,32 & 44,25 \\
\hline$S^{(2)}$ & 12,80 & 38,78 \\
\hline $\mathrm{CM}^{(3)}$ & 12,60 & 38,54 \\
\hline $\mathrm{AA}^{(4)}$ & 13,57 & 42,03 \\
\hline \multicolumn{3}{|l|}{$\mathrm{C}$} \\
\hline Com Cultivo & 13,22 & 41,71 \\
\hline \multirow[t]{2}{*}{ Sem Cultivo } & 12,93 & 40,10 \\
\hline & & \\
\hline SP & $1,50 \mathrm{NS}$ & $3,77 \mathrm{NS}$ \\
\hline $\mathrm{C}$ & $0,78 \mathrm{NS}$ & $2,02 \mathrm{NS}$ \\
\hline $\mathrm{SP} \times \mathrm{C}$ & $0,28 \mathrm{NS}$ & $0,25 \mathrm{NS}$ \\
\hline $\mathrm{CV}^{(6)} \mathrm{SP}(\%)$ & 7,94 & 9,77 \\
\hline $\mathrm{CV}^{(6)} \mathrm{C}(\%)$ & 7,22 & 7,84 \\
\hline
\end{tabular}

\section{CONCLUSÕES}

Nas camadas do solo abaixo de $10 \mathrm{~cm}$, houve sensível redução da macroporosidade com o correspondente aumento da microporosidade após quatro colheitas da cana-de-açúcar, independentemente dos sistemas de preparo do solo e cultivo da soqueira. Após esse mesmo período, houve aumento pronunciado na densidade do solo para as camadas compreendidas entre 20 e $50 \mathrm{~cm}$, independentemente do manejo do solo.

Os sistemas de preparo do solo, cultivo da soqueira e épocas de amostragem não revelaram mudanças consistentes na densidade do solo.

O cultivo da soqueira favoreceu o aumento da macroporosidade com subseqüente decréscimo na microporosidade, sendo o efeito inverso observado após a colheita.

Os sistemas de preparo do solo e de cultivo da soqueira não afetaram o perfilhamento e tampouco a produtividade da cultura. 


\section{REFERÊNCIAS}

BARBIERI, J.L.; ALLEONI, L.R.F.; DONZELLI, J.L. Avaliação agronômica e econômica de sistemas de preparo e solo para cana-de-açúcar. Revista Brasileira de Ciência do Solo, Campinas, v.21, n.1, p.89-98, 1997.

BORGES, E.N.; LOMBARDI NETO, F.; CORREAA, G.F.; BORGES, E.V.S.; Alterações físicas introduzidas por diferentes níveis de compactação em Latossolo Vermelho-Escuro textura média. Pesquisa Agropecuária Brasileira, Brasília, v.34, n.9, p.1663-7, 1999.

CASAGRANDE, A.A. Cultivo mecânico e adubação na soqueira da cana-de-açúcar - efeitos na planta e no solo. 1973.99 f. Tese (Doutorado em Ciências) - Faculdade de Ciências Agrárias e Veterinárias, Universidade Estadual Paulista, Jaboticabal,1973.

CEDDIA, M.B.; ANGOS, L.H.C.; LIMA, E.; RAVELLI NETO, A.; SILVA, L.A. Sistemas de colheita da cana-de-açúcar e alterações nas propriedades físicas de um solo Podzólico Amarelo no Estado do Espírito Santo. Pesquisa Agropecuária Brasileira, Brasília, v.34, n.8, p.1467-73, 1999.

CERQUEIRA LUZ, P. H. Efeitos de sistemas de colheita e formas de cultivo de soqueira sobre a produção e qualidade tecnológica da cana-de-açúcar (Saccharum spp.). 1989. 135 f. Dissertação (Mestrado em Solos e Nutrição de Plantas) - Escola Superior de Piracicaba de Agricultura "Luiz de Queiroz", Universidade de São Paulo, Piracicaba, 1989.

DIAS, F.L.F. Sistemas de preparo de solo em área de colheita mecanizada de cana crua. $2001.83 \mathrm{f}$. Tese (Doutorado em Produção Vegetal) - Faculdade de Ciências Agrárias e Veterinárias, Universidade Estadual Paulista, Jaboticabal, 2001.

EMBRAPA. Centro Nacional de Pesquisa de Solos. Manual de métodos de análise de solos. 2.ed. Rio de Janeiro: Embrapa Produção de Informação, 1997. 212 p.

EMBRAPA. Centro Nacional de Pesquisa de Solos. Sistema brasileiro de classificação de solos. Brasília: Embrapa Produção de Informação, 1999. 412 p.

FERNANDES, J.; RIPOLI, T.C.; MILLAN, M.A. A compactação do solo e a brotação das soqueiras. Álcool \& Açúcar, São Paulo, v.3, n.12, p.12-17, 1983.

KLEIN, V.A.; LIBARDI, P.L. Densidade e distribuição do diâmetro dos poros de um Latossolo Vermelho, sob diferentes sistemas de uso e manejo. Revista Brasileira de Ciência do Solo, Viçosa, v.26, n.4, p.857-67, 2002.

GUPTA, S.C.; SHARMA, P.P.; DE FRANCHI, S.A. Compaction effects on soil structure. Advances in Agronomy, San Diego, v.42, p.311-38, 1989.

MUTTON, M.A. Efeitos de diferentes sistemas de preparo do solo na cultura da cana-de-açúcar (Saccharum spp. Var. NA 56-79). 1983. 155 f. Dissertação (Mestrado em Ciências) - Faculdade de Ciências Agrárias e Veterinárias, Universidade Estadual Paulista, Jaboticabal, 1983.

ORLANDO FILHO, J.; CARMELLO, Q.A.C.; PEXE, C.A.; GLÓRIA, A.M. Adubação de soqueiras de cana-de-açúcar sob dois tipos de despalha: cana crua x cana queimada. STAB: Açúcar e Álcool e Subprodutos, Piracicaba, v.12, n.4, p.7-11, 1994.

ORLANDO FILHO, J.; ROSSETTO, R.; MURAOK, T.; ZOTELLI. Efeitos do sistema de despalha (cana crua $\mathrm{x}$ cana queimada) sobre algumas propriedades do solo. STAB: Açúcar e Álcool e Subprodutos, Piracicaba, v.16, n.6, p.30-3, 1998.

RAIJ, B. van,; CANTARELLA, H.; QUAGGIO, J.A.; FURLANI, A.M.C. Recomendações de adubação e calagem para o Estado de São Paulo. Campinas: Instituto Agronômico, 1996. 285 p. (Boletim técnico, 100). 
SILVA, J.A.G. Comparação entre métodos expedidos para a estimativa de peso de parcelas em ensaios com cana-de-açúcar. Boletim Técnico Copersucar, Piracicaba, n.45, p.11-13, 1989.

TORMENA, C.A.; ROLOFF, G.; SÁ, J.C.M. Propriedades físicas do solo sob plantio direto influenciadas por calagem, preparo inicial e tráfego. Revista Brasileira de Ciência do Solo, Campinas, v.22, n.2, p.301-9, 1998.

VASCONCELOS, A.C.M. Desenvolvimento do sistema radicular e da parte aérea de socas de canade-açúcar sob dois sistemas de colheita: crua mecanizada e queimada manual. 2002. $140 \mathrm{f}$. Tese (Doutorado em Produção Vegetal) - Faculdade de Ciências Agrárias e Veterinárias, Universidade Estadual Paulista, Jaboticabal, 2002. 